\title{
Fusion of Continuous-Valued Sensor Measurements using Confidence-Weighted Averaging
}

\author{
Wilfried Elmenreich \\ Vienna University of Technology \\ Institute of Computer Engineering \\ Vienna, Austria \\ wil@vmars. tuwien.ac.at
}

Accepted for publication at Journal of Vibration and Control

\begin{abstract}
This paper presents a method for fusing measurement samples from multiple sensors into a dependable robust estimation of a variable in the control environment. Each sensor measurement is represented by a measurement value and a confidence marker that corresponds to the respective variance of the measurement. We propose the ConfidenceWeighted Averaging (CWA) algorithm for fusing measurements with respect to the estimated variance of the measurement error. For calibrated sensors with uncorrelated error functions this algorithm is optimal for producing a result with minimum mean squared error.
\end{abstract}

\section{Introduction}

Due to the availability of cheap sensing elements and larger, integrated systems, the number of sensor data sources in typical embedded applications will increase in the future. For example, the DECOS integrated architecture [1] proposes a concept where sensor data from different distributed application subsystems in an automobile is made available to other subsystems via a virtual gateway concept. Furthermore, the advent of sensor networks in the cabled and wireless domain makes it possible to easily instrument a large number of sensors. This allows applications to take advantage of more sensor information about the environment, however requires means to systematically combine sensor information from sensors with different accuracy and reliability. The fused result should be more exact and more dependable than the single sensor measurements.

In this paper we focus on the problem of fusing a sample of several continuous-valued sensor measurements into a more robust and more accurate estimation of the measurand using a statistical approach. Since we do not assume to have a model of the observed process, we do not regard previous measurements (series), but only concurrent measurements from the same real-time entity. By taking advantage of the smart transducer concept [2], we can expect each measurement to be pre-calibrated and assigned with a confidence marker that gives an estimation of the quality of the measurement. Our fusion algorithm combines the measurements with respect to their variance into a more accurate estimation of the measurand and gives an estimation of the result's confidence.

The rest of the paper is structured as follows: Section 2 describes the fusion problem to be solved. Section 3 gives an overview on related work. Section 4 elaborates on 
a representation of confidence in a digital format. The algorithm and its analysis is presented in Section 5. Section 6 presents experimental results from a multi-sensor case study. The paper is concluded in Section 7.

\section{The Fusion Problem}

Given is a set of sensors that measure the same real-time entity in the process environment. We assume the sensors to be calibrated $^{1}$, so that the measurement errors are only of stochastic nature. Furthermore, the correlation between the sensor's error function needs to be insignificant. We will show in Section 6 that these assumptions hold for real sensor networks.

Analyses of real sensors have shown that it is difficult to make any assumption on the error distribution of a sensor. Figure 1 shows the distribution of measurement errors that has been measured for a particular distance sensor. Therefore, we assume that the probability distribution function of the sensors' measurement errors is uncharacterized.

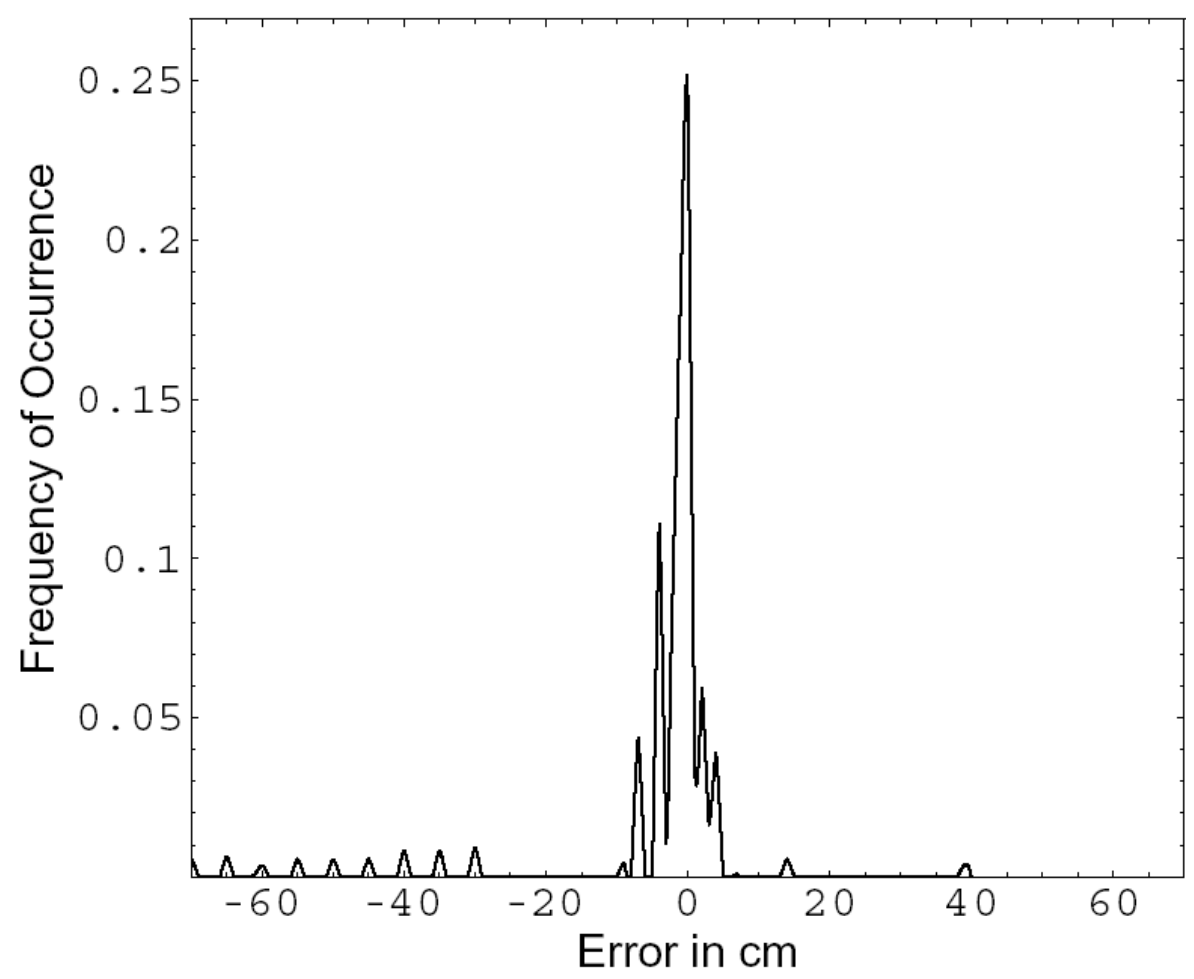

Figure 1: Error histogram for Sharp GP2D02 infrared sensor (from [3])

The sensors will produce a sample of observations, where an observation consists of the measurement value, the measurement instant, a confidence marker and the respective name of the measured entity.

Furthermore we assume that the observations are taken synchronuously within a time window that is sufficiently small so that the variable to be measured does not change significantly within that interval.

\footnotetext{
${ }^{1}$ Note that there are cases, where a systematic error cannot be removed by calibration, e. g., when values beyond the sensor's measurement range are mapped to a default value.
} 


\section{Related Work}

In the literature, several methods can be found for classifier fusion or decision fusion based on sensor information. Some examples are voting mechanisms [4], based on reliability of each sensor or classifier ([5]) or, as in [6], also considering correlations between the sources. Other more complex methods include Hidden Markov Models or Neural Networks (e.g. [7]), all with the intention to minimize the expected error of the fused result.

Focusing at fusion of continuous-valued sensor measurements, the fault-tolerant sensor averaging algorithm proposed by Marzullo in [8], is closely related to our approach. Unlike the Kalman filter [9], Marzullo's approach is stateless, thus does not require data from previous measurements in the fusion process. We will compare the results from our Confidence-Weighted Averaging (CWA) algorithm to the fault-tolerant sensor averaging algorithm in Section 6.

A scheme for confidence markers in digital systems is presented by Parhami in [10]. The proposed approach attaches so-called dependability tags to each data object and updates these tags according to operations performed on these data objects.

Another idea that contributed to the work in this paper is given by sensor validation for fieldbus nodes. So-called self-validating sensors are able to provide a standardized digital signal and generate diagnostic information. In the Oxford SEVA system [11], each measurement is delivered as a validated value, together with the validated uncertainty and a measurement value status.

\section{Representation of Confidence Markers}

The confidence measure will be introduced as an integer value between 0 and $\operatorname{conf}_{\max }$, where 0 is defined to be the lowest confidence and conf $f_{\max }$ is the highest confidence.

We have chosen the statistical variance as a reciprocal measure for confidence. Also, the ISO Guide to the Expression of Uncertainty in Measurement [12] suggests statistical variance as a measure for uncertainty.

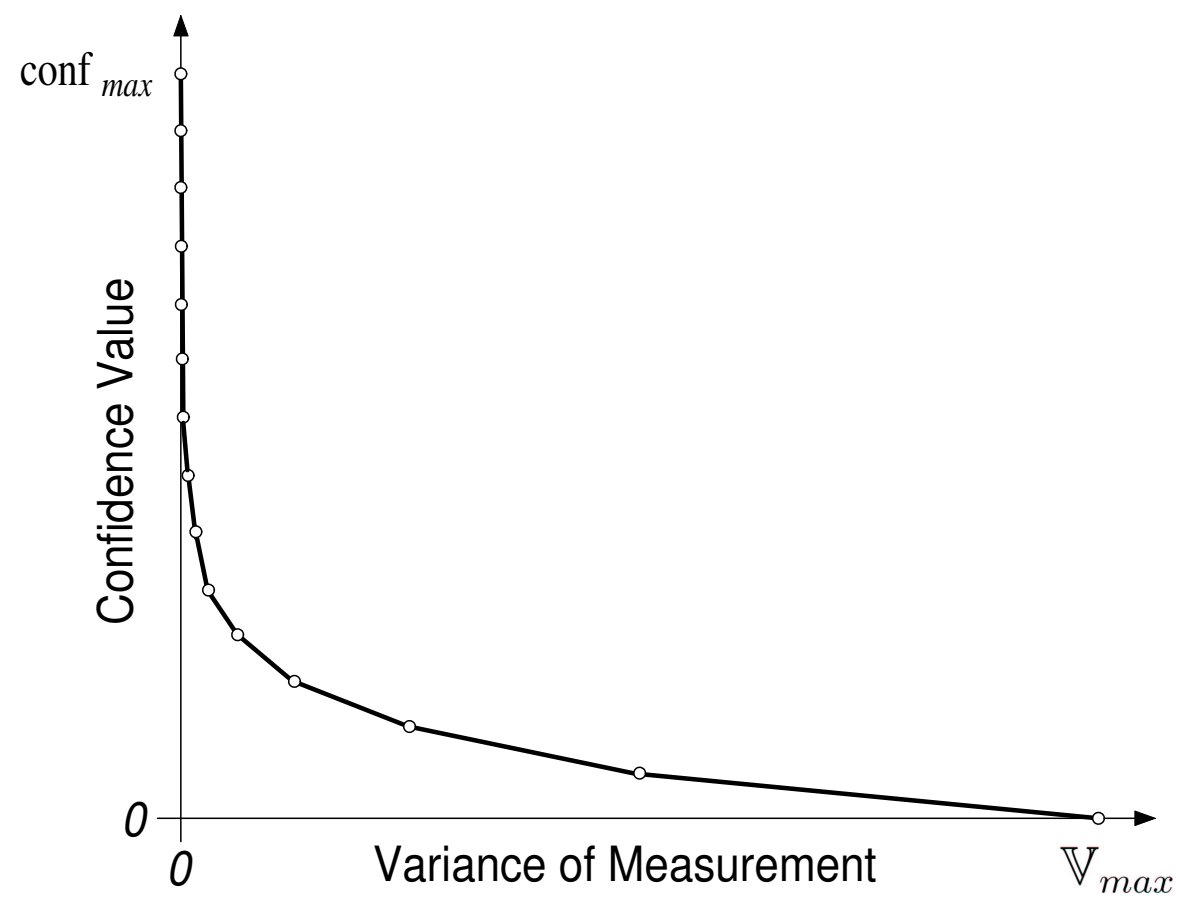

Figure 2: Conversion function for confidence/variance values (based on a logarithmic scale) 
In order to enable operations based on the confidence of observations from different sources, the confidence has to be standardized. We assume, that in the best case, variance will be close to 0 , thus corresponding to the maximum confidence. In the worst case, a sensor will deliver a random value within its measurement range for the measurement. The worst-case variance can thus be calculated as the variance of a uniformly distributed random function between the limits $a$ and $b$ :

$$
\mathbb{V}[S]=\frac{(b-a)^{2}}{12}
$$

where $\mathrm{a}$ and $\mathrm{b}$ are the minimum and maximum values of the expected uniformly distributed random function. It is possible to find a probability distribution function that produces even greater variances, however we assume that all measurements with variances of $\mathbb{V}_{\max }$ or greater are nearly useless and therefore are mapped into the same class of minimum confidence.

A standard message format as it is used in the TTP/A sensor bus protocol [13] maps the measurement values to values between 0 and 200. If the sensor measurement is totally random, the variance can be calculated according to equation 1 . This worst case $\mathbb{V}[S]$ equals $\frac{200^{2}}{12}$ or 3333.33 . On the other hand, a variance of 0 corresponds to best possible case. Without restrictions to generality we have elaborated a mapping between a small number of confidence values and the respective variances.

Using a linear transformation between confidence values and variance would not be feasible, since the variances that indicate exact measurements are of greater interest than those measurements with large variances. Therefore, we decided to use a logarithmic scale to define the confidence values between $\min _{\text {conf }}$ and $\max _{\text {conf }}$ (see figure 2 ). Due to the expected computational load when doing logarithmic and exponential operations on embedded systems, we suggest the implementation of look-up tables for the conversion from confidence value to variance. Table 1 depicts a conversion table for 16 different levels of confidence.

\begin{tabular}{|c|c|c|}
\hline Confidence value & $\begin{array}{c}\text { Interval for uniformly } \\
\text { distributed error }\end{array}$ & Statistical Variance \\
\hline \hline 0 & {$[-100.0,100.0]$} & 3333.33 \\
\hline 1 & {$[-70.2,70.2]$} & 1644.65 \\
\hline 2 & {$[-49.3,49.3]$} & 811.47 \\
\hline 3 & {$[-34.7,34.7]$} & 400.37 \\
\hline 4 & {$[-24.3,24.3]$} & 197.54 \\
\hline 5 & {$[-17.1,17.1]$} & 97.47 \\
\hline 6 & {$[-12.0,12.0]$} & 48.09 \\
\hline 7 & {$[-8.4,8.4]$} & 23.73 \\
\hline 8 & {$[-5.9,5.9]$} & 11.71 \\
\hline 9 & {$[-4.2,4.2]$} & 5.78 \\
\hline 10 & {$[-2.9,2.9]$} & 2.85 \\
\hline 11 & {$[-2.1,2.1]$} & 1.41 \\
\hline 12 & {$[-1.4,1.4]$} & 0.69 \\
\hline 13 & {$[-1.0,1.0]$} & 0.34 \\
\hline 14 & {$[-0.7,0.7]$} & 0.17 \\
\hline 15 & & 0.08 \\
\hline
\end{tabular}

Table 1: Conversion table for 16 different levels of confidence 


\section{Confidence-Weighted Averaging}

We suggest an algorithm for fusing data from replicated sensors based on weighted averages. The fused value $x_{F U S E D}$ is calculated as the weighted average of all measurement $x_{i}$, the weights $w_{i}$ being derived from the reciprocal of the variance of each sensor $S_{i}$.

$$
x_{F U S E D}=\sum_{i=1}^{n} x_{i} w_{i}
$$

with

$$
w_{i}=\frac{1}{\mathbb{V}\left(S_{i}\right) \sum_{j=1}^{n} \frac{1}{\mathbb{V}\left(S_{j}\right)}}
$$

where $n$ is the number of observations, $x_{i}$ represents a measurement taken by sensor $S_{i}$ and $\mathbb{V}\left(S_{i}\right)$ is the estimated variance associated to that sensor. Under the assumption of independence of errors between sensors and supposing that the expected error $\mathbb{E}\left[x_{i}-x\right]$ is equal to 0 , this method minimizes the expected variance of the fused value.

Proof. $X_{F U S E D}$ is a weighted average of $i$ independent random variables $X_{i}$. The weights $w_{i}$ should be chosen so that they minimize the mean squared error of the fused variable $X_{F U S E D}$. Furthermore, we require that the fused estimate is unbiased, that is that the average deviation from the true measurement $X$ is equal to 0 .

$$
\mathbb{E}\left[X_{F U S E D}-X\right]=\mathbb{E}\left[\sum_{i=1}^{n} w_{i} x_{i}-x\right]=0
$$

The expected squared error of the fused result can be expressed as

$$
\mathbb{E}\left[\left(X_{F U S E D}-X\right)^{2}\right]=\sigma_{F U S E D}^{2}=\sum_{i=1}^{n} w_{i}^{2} \sigma_{i}^{2} .
$$

Given that $\mathbb{E}\left[x_{i}-x\right]=0$ and $\mathbb{E}[x]=x$ we deduce that $\sum_{i=1}^{n} w_{i}=1$. Looking for the weights $w_{i}$ that minimize the expression in 5 , we substitute $w_{1}=1-\sum_{j=2}^{n} w_{j}$ and calculate the partial derivative for each weight:

$$
\frac{\partial \sigma_{F U S E D}^{2}}{\partial w_{i}}=-2 \sigma_{1}^{2}\left(1-\sum_{j=2}^{n} w_{j}\right)+2 w_{i} \sigma_{i}^{2}=0
$$

Setting all partial derivatives equal we can derive the expression

$$
\sigma_{1}^{2}\left(1-\sum_{j=2}^{n} w_{j}\right)=w_{2} \sigma_{2}^{2}=\ldots=w_{n} \sigma_{n}^{2}
$$

We see that all weights $w_{i}, i=2 \ldots n$ are proportional to the reciprocal of the corresponding $\sigma_{i}^{2}$. We can therefore express them as

$$
w_{i}=\xi / \sigma_{i}^{2}
$$

and receive the expression

$$
\sigma_{1}^{2}\left(1-\sum_{j=2}^{n} \frac{\xi}{\sigma_{j}^{2}}\right)=\frac{\xi}{\sigma_{2}^{2}} \sigma_{2}^{2}=\ldots=\frac{\xi}{\sigma_{n}^{2}} \sigma_{n}^{2}
$$

We can now solve for $\xi$ as follows: 


$$
\begin{gathered}
\sigma_{1}^{2}\left(1-\sum_{j=2}^{n} \frac{\xi}{\sigma_{j}^{2}}\right)=\xi \\
1-\sum_{j=2}^{n} \frac{\xi}{\sigma_{j}^{2}}=\frac{\xi}{\sigma_{1}^{2}} \\
1=\frac{\xi}{\sigma_{1}^{2}}+\sum_{j=2}^{n} \frac{\xi}{\sigma_{j}^{2}} \\
1=\xi \sum_{j=1}^{n} \frac{1}{\sigma_{j}^{2}} \\
\xi=\frac{1}{\sum_{j=1}^{n} \frac{1}{\sigma_{j}^{2}}}
\end{gathered}
$$

Substituting 14 into 8 we receive 15 as the optimal weight for each $x_{i}$

$$
w_{i}=\frac{1}{\sigma_{i}^{2} \sum_{j=1}^{n} \frac{1}{\sigma_{j}^{2}}}
$$

To ensure that the solution is in fact a minimum, we derive the second partial derivative which is greater than 0 , since $\sigma_{1}^{2}$ and $\sigma_{2}^{2}$ are in all cases greater than 0 :

$$
\frac{\partial^{2} \sigma_{F U S E D}^{2}}{\partial w_{i}^{2}}=2 \sigma_{1}^{2}+2 \sigma_{i}^{2}>0
$$

The formula for calculating the fused value $x_{F U S E D}$ is therefore

$$
x_{F U S E D}=\frac{\sum_{i=1}^{n} \frac{x_{i}}{\mathbb{V}\left(S_{i}\right)}}{\sum_{i=1}^{n} \frac{1}{\mathbb{V}\left(S_{i}\right)}}
$$

The variance of $X_{F U S E D}$ being always smaller than any of the input variances and is derived as follows:

$$
\sigma_{F U S E D}^{2}=\sum_{i=1}^{n} w_{i}^{2} \sigma_{i}^{2}=\sum_{i=1}^{n} \frac{\sigma_{i}^{2}}{\sigma_{i}^{4}\left(\sum_{j=1}^{n} \frac{1}{\sigma_{j}^{2}}\right)^{2}}=\frac{\sum_{i=1}^{n} \frac{1}{\sigma_{i}^{2}}}{\left(\sum_{i=1}^{n} \frac{1}{\sigma_{i}^{2}}\right)^{2}}=\frac{1}{\sum_{i=1}^{n} \frac{1}{\sigma_{i}^{2}}}
$$

The fused variance of the fusion result, which can be interpreted as a virtual sensor $S_{F U S E D}$ is thus

$$
\mathbb{V}\left(S_{F U S E D}\right)=\frac{1}{\sum_{i=1}^{n} \frac{1}{\mathbb{V}\left(S_{i}\right)}}
$$

This method is optimal in the sense that it minimizes the expected variance of the fused result. 


\section{$6 \quad$ Experimental Results}

To evaluate the practical applicability of CWA, we have applied the algorithm to data from three infrared sensors of type Sharp GP2D02 and two Polaroid 6500 series ultrasonic sensors.

The infrared sensors are designed for measuring distances within the range of $10-80 \mathrm{~cm}$. They show problematic behavior when there is no object within detection range which is as far as about $110 \mathrm{~cm}$. In this case the returned data is unreliable and may correspond to arbitrary measurements within the range. To detect such erroneous measurements a filtering algorithm was applied locally at each sensor. The filter considers four subsequent measurements of a sensor and determines that there is no object within range if the jitter of these measurements is larger than a particular threshold.

Table 2 shows the results of the fusion with the CWA algorithm. The first column indicates which sensor sources have been used for the fusion and if the above described filtering has been applied to the IR sensors. The next three columns contain the error and variance of the fused result.

For comparison we have fused the same data set with the the fault-tolerant sensor averaging algorithm proposed by Marzullo [8].

In Marzullo's algorithm each sensor measurement is modeled by an interval that is expected to contain the real sensor measurement. If a sensor delivers a measurement with the real value outside this interval, the sensor is considered faulty. It is required to parametrize the expected number of faulty sensors at once as $t$.

Since the behavior of the measurement errors of the employed sensors makes it difficult to select the best $t$ a priori for a given configuration we have performed multiple runs of the fault-tolerant sensor averaging algorithm for each possible $t$. Table 3 lists the results obtained from the different runs using various sensor configurations.

In comparison to the results from the CWA algorithm, the performance is similar for homogeneous sensor configurations while CWA performs much better for heterogeneous sensor configurations. Especially for the homogeneous sensor configurations the sensor

\begin{tabular}{|l|c|c|c|}
\hline Fusion sources & $\begin{array}{c}\text { Mean squar- } \\
\text { ed error } \\
\left(\mathrm{cm}^{2}\right)\end{array}$ & $\begin{array}{c}\text { Mean abso- } \\
\text { lute error } \\
(\mathrm{cm})\end{array}$ & $\begin{array}{c}\text { Estimated } \\
\text { variance } \\
\left(\mathrm{cm}^{2}\right)\end{array}$ \\
\hline \hline US 1 + US 2 & 9.29 & 1.52 & 8.54 \\
\hline $\begin{array}{l}\text { IR 1 + IR 2 + IR 3 } \\
\text { (unfiltered) }\end{array}$ & 129.00 & 7.29 & 119.52 \\
\hline $\begin{array}{l}\text { US 1 + IR 1 } \\
\text { (unfiltered) }\end{array}$ & 6.41 & 1.66 & 6.96 \\
\hline $\begin{array}{l}\text { US 1 + IR 1 } \\
\text { (filtered) }\end{array}$ & 55.97 & 1.63 & 6.56 \\
\hline $\begin{array}{l}\text { IR 3 + IR 2 + IR 3 } \\
\text { (filtered) }\end{array}$ & 6.65 & 1.37 & 6.14 \\
\hline $\begin{array}{l}\text { US 1+US 2+IR 1+IR 2+ } \\
\text { +IR 3 (unfiltered) }\end{array}$ & 5.32 & 1.31 & 4.87 \\
\hline $\begin{array}{l}\text { US 1+US 2+IR 1+IR 2+ } \\
\text { (filtered) }\end{array}$ & & & \\
\hline
\end{tabular}

Table 2: Performance of the CWA algorithm for the examined sensor configurations 


\begin{tabular}{|l|c|c|c|c|}
\hline Fusion sources & $t$ & $\begin{array}{c}\text { Mean squar- } \\
\text { ed error } \\
\left(\mathrm{cm}^{2}\right)\end{array}$ & $\begin{array}{c}\text { Mean abso- } \\
\text { lute error } \\
(\mathrm{cm})\end{array}$ & $\begin{array}{c}\text { Estimated } \\
\text { variance } \\
\left(\mathrm{cm}^{2}\right)\end{array}$ \\
\hline \hline US 1 + US 2 & $\mathbf{0}$ & $\mathbf{1 0 . 0 2}$ & $\mathbf{1 . 9 9}$ & $\mathbf{9 . 5 7}$ \\
& 1 & 10.99 & 2.08 & 10.65 \\
\hline IR 1+IR 2+IR 3 & 0 & 477.09 & 10.60 & 430.41 \\
(unfiltered) & $\mathbf{1}$ & $\mathbf{1 3 0 . 6 3}$ & $\mathbf{7 . 3 9}$ & $\mathbf{1 1 3 . 7 7}$ \\
& 2 & 190.63 & 10.51 & 180.11 \\
\hline IR 1+IR 2+IR 3 & 0 & 2061.90 & 26.25 & 1492.08 \\
(filtered) & $\mathbf{1}$ & $\mathbf{8 2 . 9 5}$ & $\mathbf{6 . 7 2}$ & $\mathbf{7 6 . 8 7}$ \\
& 2 & 100.67 & 7.33 & 90.49 \\
\hline US 1+ IR 1 & 0 & 1129.60 & 14.32 & 986.78 \\
(unfiltered) & $\mathbf{1}$ & $\mathbf{2 1 2 . 3 5}$ & $\mathbf{1 0 . 8 7}$ & $\mathbf{1 7 3 . 7 1}$ \\
\hline US 1 + IR 1 & 0 & 1300.40 & 16.74 & 1092.96 \\
(filtered) & $\mathbf{1}$ & $\mathbf{2 1 2 . 0 8}$ & $\mathbf{1 1 . 1 8}$ & $\mathbf{1 8 1 . 7 4}$ \\
\hline US 1 + US 2 + & 0 & 1646.96 & 18.84 & 1376.00 \\
+IR 1+IR 2+IR 3 & 1 & 260.00 & 3.96 & 257.69 \\
(unfiltered) & $\mathbf{2}$ & $\mathbf{4 8 . 2 5}$ & $\mathbf{4 . 5 7}$ & $\mathbf{4 5 . 5 5}$ \\
& 3 & 117.55 & 7.21 & 101.87 \\
& 4 & 190.63 & 10.51 & 180.11 \\
\hline US 1 + US 2 + & 0 & 2387.56 & 28.88 & 1680.39 \\
+IR 1+IR 2+IR 3 & 1 & 139.74 & 3.45 & 138.99 \\
(filtered) & $\mathbf{2}$ & $\mathbf{1 2 . 2 1}$ & $\mathbf{2 . 4 9}$ & $\mathbf{1 1 . 8 6}$ \\
& 3 & 70.08 & 6.44 & 63.15 \\
& 4 & 100.67 & 7.33 & 90.49 \\
\hline
\end{tabular}

Table 3: Performance of Marzullo's fault-tolerant sensor averaging algorithm for the examined sensor configurations. $t$ represents the number of faulty sensors to be tolerated

errors turned out to be stronger correlated which affected the quality of the fused result. Nevertheless, the CWA algorithm delivers a serviceable result of reduced quality.

\section{Conclusion and Outlook}

We have proposed an algorithm for fusing measurement samples from multiple sensors into a dependable robust estimation of a variable in the control environment. This ConfidenceWeighted Averaging (CWA) algorithm takes values annotated with confidence markers as inputs and output. The confidence marker corresponds to the respective variance of the value. We have shown that this algorithm is optimal for producing the minimum possible variance of the average result for calibrated sensors with uncorrelated error functions.

However, CWA is based on the independence of measurement errors, an assumption that cannot generally be made in sensor fusion applications. In the future work we will extend our algorithm by taking correlated error functions into account. Thus, we expect a more accurate estimation of the results quality for fusing measurements taken by the same type of sensors. 


\section{Acknowledgments}

This work was supported by the Austrian FWF project TTCAR under contract No. P18060-N04

\section{References}

[1] H. Kopetz, R. Obermaisser, P. Peti, and N. Suri. From a federated to an integrated architecture for dependable embedded systems. Research Report 22/2004, Technische Universität Wien, Institut für Technische Informatik, Vienna, Austria, 2004.

[2] W. Elmenreich and S. Pitzek. Smart transducers - principles, communications, and configuration. In Proceedings of the 7th IEEE International Conference on Intelligent Engineering Systems, volume 2, pages 510-515, Assuit - Luxor, Egypt, March 2003.

[3] W. Elmenreich. Sensor Fusion in Time-Triggered Systems. PhD thesis, Technische Universität Wien, Institut für Technische Informatik, Vienna, Austria, 2002.

[4] B. Parhami. Optimal algorithms for exact, inexact, and approval voting. TwentySecond International Symposium on Fault-Tolerant Computing, 1992. FTCS-22. Digest of Papers., pages 404-411, July 1992.

[5] S.A. Aldosari and J.M.F. Moura. Fusion in sensor networks with communication constraints. In IPSN'04: Proceedings of the third international symposium on Information processing in sensor networks, pages 108-115, New York, NY, USA, 2004. ACM Press.

[6] N.S.V. Rao. A generic sensor fusion problem: Classification and function estimation. In T.Windeatt F.Roli, J.Kitler, editor, Multiple Classifier Systems: 5th International Workshop, MCS 2004. Proceedings, volume 3077 of Lecture Notes in Computer Science, pages 16-30. Springer-Verlag GmbH, January 2004.

[7] T.W.Lewis and D.M.W. Powers. Sensor fusion weighting measures in audio-visual speech recognition. In CRPIT '04: Proceedings of the 27th conference on Australasian computer science, pages 305-314, Darlinghurst, Australia, Australia, 2004. Australian Computer Society, Inc.

[8] K. Marzullo. Tolerating failures of continuous-valued sensors. ACM Transactions on Computer Systems, 8(4):284-304, November 1990.

[9] R. E. Kalman. A new approach to linear filtering and prediction problems. Transaction of the ASME, Series D, Journal of Basic Engineering, 82:35-45, March 1960.

[10] B. Parhami. A data-driven dependability assurance scheme with applications to data and design diversity. In A. Avizienis and J. C. Laprie, editors, Dependable Computing for Critical Applications, volume 4, pages 257-282. Springer Verlag, Vienna, 1991.

[11] M. Henry. Sensor validation and fieldbus. Computing \& Control Engineering Journal, 6(6):263-269, December 1995.

[12] International Organization for Standardization (ISO), Genève, Switzerland. Guide to the Expression of Uncertainty in Measurement, 1st edition, 1993.

[13] H. Kopetz et al. Specification of the TTP/A protocol. Research Report 61/2002, Technische Universität Wien, Institut für Technische Informatik, Vienna, Austria, September 2002. Version 2.00. 